\title{
Diseño de un sitio web dinámico para institución educativa
}

\section{Design of a dynamic website for educational institution}

\author{
VÁZQUEZ-DE LOS SANTOS, Laura Cristina†*, CORTES-MORALES, Griselda, VALDEZ- \\ MENCHACA, Alicia Guadalupe y MARTÍNEZ-PERALES, Diego Arnulfo
}

Universidad Autónoma de Coahuila, Facultad de Ingeniería Mecánica y Eléctrica, Unidad Norte

ID $1^{\mathrm{er}}$ Autor: Laura Cristina, Vázquez-De Los Santos / ORC ID: 0000-0002-0291-7774, Researcher ID Thomson: S-65432018, CVU CONACYT ID: 615088

ID $1^{\mathrm{er}}$ Coautor: Griselda, Cortes-Morales / ORC ID: 0000-0002-2567-7056, CVU CONACYT ID: 617827

ID $2^{\text {do }}$ Coautor: Alicia Guadalupe, Valdez-Menchaca / ORC ID: 0000-0002-3494-4830, Researcher ID Thomson: S-45512018, CVU CONACYT: 292172

ID $3^{\text {do }}$ Coautor: Diego Arnulfo, Martínez-Perales / CVU CONACYT ID: 1015619

DOI: $10.35429 / J I T .2019 .21 .6 .20 .25$

Recibido: 10 de Agosto, 2019; Aceptado 30 de Diciembre, 2019

\section{Resumen}

El objetivo de este artículo es diseñar un sitio web para una institución educativa con un modelo de datos dinámico que permita agregar, editar y actualizar la información de manera fácil. En la metodología, se utilizarán conceptos de la ingeniería de sistemas durante el proceso de desarrollo de sistemas, documentando cada etapa. Llevando a cabo las etapas de análisis de requerimientos y de diseño de modelo de datos, considerando las partes involucradas. Se diseñó el modelo Entidad Relación con el propósito de confirmar las necesidades lógicas de la información. Además, se creó el modelo relacional, donde se detallan los atributos de cada entidad. Como sistema manejador de base de datos se utilizó MySQL. Parte del diseño del modelo de datos incluye la manera en que se interactúa con el mismo, para esto se utiliza el sistema CRUD. Con el diseño de los modelos de datos: modelos lógicos y de base de datos, se creó el script para la creación de la base de datos dinámica, la cual se utilizará para almacenar toda la información pertinente a la institución educativa. Como resultado en el sitio web se garantizó la correcta funcionalidad de la base de datos.

Modelo de datos dinámico, Sitio web, Modelo de datos

\begin{abstract}
The objective of this article is to design a website for an educational institution with a dynamic data model that allows you to easily add, edit and update information. In the methodology, systems engineering concepts will be used during the system development process, documenting each stage. Carrying out the stages of requirements analysis and data model design, considering the parties involved. The Entity Relationship model was designed with the purpose of confirming the logical needs of the information. In addition, the relational model was created, where the attributes of each entity are detailed. MySQL was used as the database management system. Part of the design of the data model includes the way in which it interacts with it, for this the CRUD system is used. With the design of the data models: logical and database models, the script for the creation of the dynamic database was created, which will be used to store all the information relevant to the educational institution. As a result, the correct functionality of the database was guaranteed on the website.
\end{abstract}

Dynamic data model, Website, Data model

Citación: VÁZQUEZ-DE LOS SANTOS, Laura Cristina, CORTES-MORALES, Griselda, VALDEZ-MENCHACA, Alicia Guadalupe y MARTÍNEZ-PERALES, Diego Arnulfo. Diseño de un sitio web dinámico para institución educativa. Revista de Tecnologías de la Información. 2019. 6-21: 20-25

\footnotetext{
* Correspondencia del Autor (laura_vazquez@uadec.edu.mx)

$\dagger$ Investigador contribuyendo como primer autor.
} 


\section{Introducción}

Hoy en día para los negocios e instituciones es muy importante contar con un sitio web que les permita estar visibles o tener presencia de forma global. Lo anterior además ofrece respuestas a las necesidades del personal involucrado.

En las instituciones educativas los sitios web son importantes debido a que permiten entregar a sus estudiantes, trabajadores y aspirantes información de forma eficiente. Aunque esta función se puede llevar a cabo por distintos medios, por ejemplo redes sociales.

El adecuado diseño y funcionalidad de un sistema web da un valor agregado a la organización.

El objetivo de este artículo es diseñar un sitio web para una institución educativa con un modelo de datos dinámico que permita agregar, editar y actualizar la información de manera fácil.

\section{Desarrollo de Secciones}

En este artículo se muestra a detalle el diseño de un sitio web dinámico para una institución educativa. El diseño web dinámico permitirá manipular la información dentro del sitio. Al presentar un diseño como el mencionado, se podrá distribuir la información variada y que proviene de múltiples fuentes.

\section{Metodología a desarrollar}

Como se mencionó anteriormente un problema a resolver es que el sitio web permita difundir información de manera continua y de esta forma reducir la necesidad de dar mantenimiento al sitio.

Considerando el análisis como parte del desarrollo de sitio web, se consideraron los antecedentes de una institución educativa. El sitio actual cuenta con información esencial para los alumnos y docentes, pero para llevar a cabo ediciones se requiere modificar el propio código de la página web, situación que se ve complicada.
Con esta necesidad se diseña un sistema que mediante una base de datos dinámica tendrá las dos funciones que se busca tener en el sitio: adición de información y edición de la misma, además de realizar respaldos de información, seguridad de la información, etc.

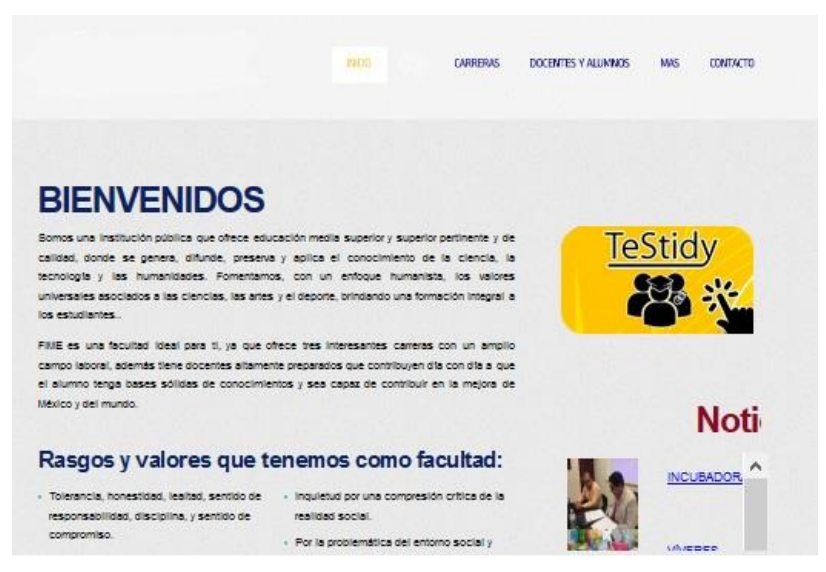

Figura 1 Sitio web actual de la institución educative Fuente: Elaboración Propia

Dentro de la etapa de análisis se tiene la necesidad de incorporar ciertos aspectos de las redes sociales dentro del sitio web, como lo son las funciones de generar comentarios en las publicaciones, generar notificaciones de eventos o noticias relevantes, la creación de galerías de imágenes, la creación de "tags" para identificar los temas principales de las publicaciones, la posibilidad de impedir la publicación de comentarios y la posibilidad de permitir a usuarios hacer publicaciones de ciertos temas.

Tomando la información recopilada a partir del análisis de requerimientos se procede a la fase de diseño. Para hacer uso de las bases de datos dinámicas se requiere generar un modelo de datos, para esto se realizaron los siguientes diagramas para confirmar que se cumplan los requerimientos.

\section{Diagrama Entidad Relación}

El diagrama E-R se realizó con el propósito de confirmar las necesidades lógicas de la información que genera la institución. La siguiente figura muestra a detalla dicho diagrama. 


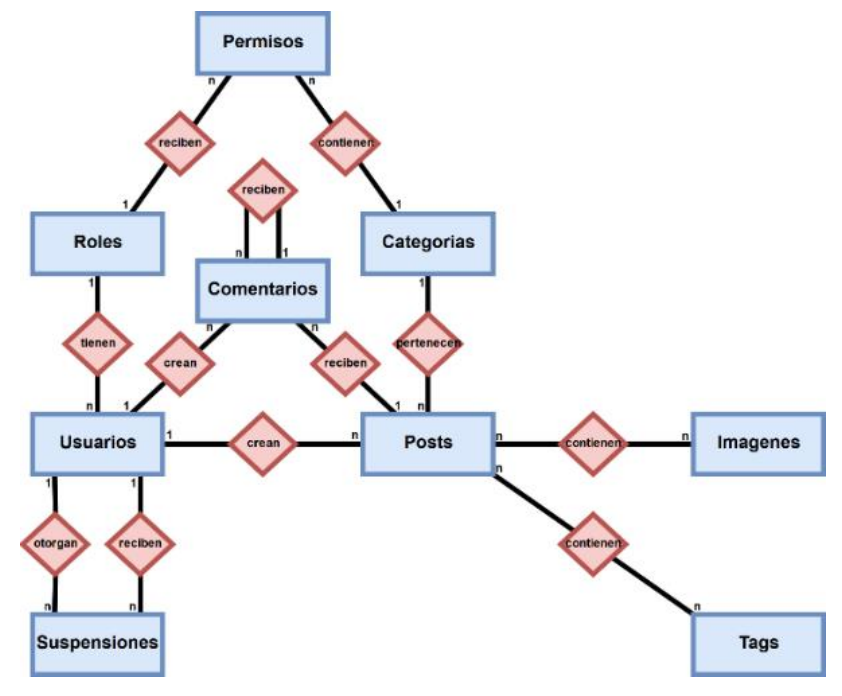

Figura 2 Diagrama Entidad Relación Fuente: Elaboración Propia

Mediante la abstracción, se encontró que para facilitar la difusión de información era necesario abarcar todo tipo de información que se desee publicar en una sola entidad, llamada "Posts" (publicaciones). Para mejor entender este concepto se utilizó la analogía de un boletín físico que se adhiere a un panel de corcho utilizando chinchetas.

De esta manera se pueden describir lógicamente las entidades más importantes del diseño:

- $\quad$ Posts: Los posts son publicaciones que contienen la información importante que se desea difundir, estos pueden contener imágenes de ser necesario, además de tags para facilitar su búsqueda. Todo post pertenece a una categoría que define el tipo de información que contiene el post (Por ejemplo: Noticia, Evento). Los posts son creados por usuarios con el rol adecuado, además cualquier usuario puede comentar en los posts y subsecuentemente se puede comentar los comentarios.

Usuarios: Los usuarios tienen roles (Por ejemplo: Alumno, Docente, Administrativo), estos roles reciben permisos que les permiten crear posts de cierta categoría. Todo usuario puede comentar los posts y a manera de moderación los usuarios con roles superiores son capaces de otorgar suspensiones a otros usuarios.
Con estas entidades y sus relaciones se satisfacen las necesidades principales que se presentaron inicialmente.

Diagrama relacional de base de datos

La siguiente figura muestra el modelo Relacional construido a partir del modelo Entidad Relación.

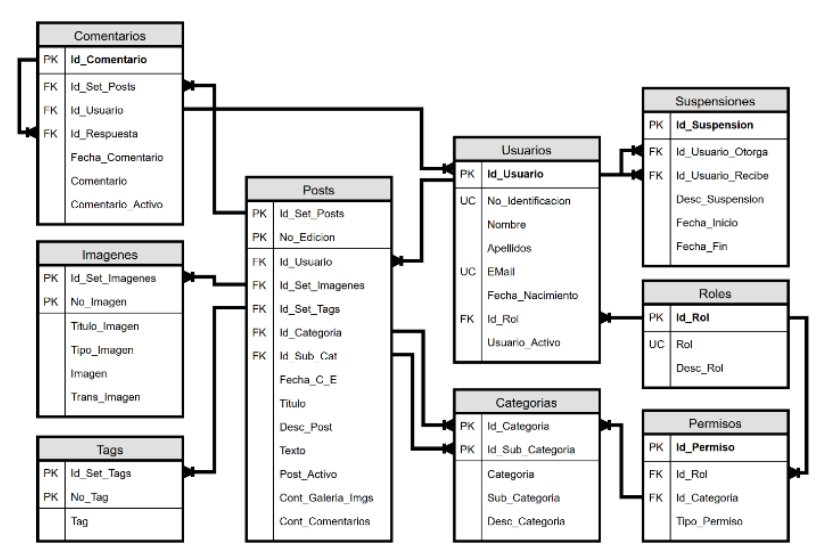

Figura 3 Modelo Relacional Fuente: Elaboración Propia

En este modelo se detallan los atributos de cada entidad.

Posts:

- Id_Set_Posts: Llave primaria, número de identificación de una publicación.

- $\quad$ No_Edicion: Llave primaria, número de edición de la publicación.

- Id_Usuario: Llave foránea, corresponde al usuario que creo la publicación, o al usuario que edito la publicación si el número de edición es mayor a 1 .

- Id_Set_Imagenes: Llave foránea, corresponde al set de imágenes utilizado en la publicación.

- Id_Categoria: Llave foránea, corresponde a la categoría de la publicación.

- Id_Sub_Cat: Llave foránea, corresponde a la subcategoría de la publicación.

Fecha_C_E: Fecha de creación de la publicación, o fecha de edición si el número de edición es mayor a 1.

- Titulo: Título de la publicación.

- Desc_Post: Descripción corta de la publicación.

- $\quad$ Texto: Texto de la publicación.

- Post_Activo: Determina el estado de la publicación (activo/inactivo).

Cont_Galeria_Imgs: Determina si la publicación hace uso de galería de imágenes. 
- Cont Comentarios: Determina si la publicación acepta comentarios.

Usuarios:

- Id_Usuario: Llave primaria, número de identificación de un usuario.

- No_Identificacion: Dato único, número de identificación otorgado por la Facultad (ej. Numero de matrícula).

- $\quad$ Nombre: Nombre del usuario.

- $\quad$ Apellidos: Apellidos del usuario.

- $\quad$ Email: Dato único, dirección de correo del usuario.

- $\quad$ Fecha_Nacimiento: Fecha de nacimiento del usuario.

Id_Rol: Llave foránea, rol que desempeña el usuario.

Usuario_Activo: Determina si el usuario puede hacer uso de las funciones del sitio.

\section{Categorías:}

- Id_Categoria: Llave primaria, número de identificación de una categoría.

- Id_Sub_Categoria: Llave primaria, número de identificación de una subcategoría.

- Categoria: Nombre de la categoría.

- Sub_Categoria: Nombre de la subcategoría.

Desc_Categoria: Descripción de la categoría/subcategoría.

Imágenes:

- Id_Set_Imagenes: Llave primaria, número de identificación de un set de imágenes.

- No_Imagen: Llave primaria, número de identificación de una imagen dentro de un set de imágenes.

- Titulo_Imagen: Titulo de la imagen.

- Tipo_Imagen: Tipo de la imagen (ej. png, jpg, gif).

Imagen: Imagen convertida en formato almacenable.

Trans_Imagen: Imagen transformada para uso variado, por lo general miniaturas de la imagen original.

Tags:

- Id_Set_Tags: Llave primaria, número de identificación de un set de tags.

No_Tag: Llave primaria, número de identificación de un tag dentro de un set de tags.

Tag: Palabra relacionada al contenido del post.

\section{Comentarios:}

- Id_Comentario: Llave primaria, número de identificación de un comentario.

- Id_Set_Posts: Llave foránea, corresponde al post en el cual se realizó el comentario.

- Id_Usuario: Llave foránea, corresponde al usuario que creo el comentario.

- Id_Respuesta: Llave foránea, corresponde al comentario al que se esta respondiendo, esto si el comentario se realizó en respuesta a otro comentario.

- Fecha_Comentario: Fecha en que se realizó el comentario.

- Comentario_Activo: Determina si el comentario es visible a los demás usuarios.

Roles:

- Id_Rol: Llave primaria, número de identificación del rol.

- Rol: Dato único, nombre del rol (ej. alumno, docente, administrativo).

- Desc_Rol: Descripción del rol.

Permisos:

- Id_Permiso: Llave primaria, número de identificación de un permiso.

- $\quad$ Id_Rol: Llave foránea, corresponde al rol al que se está asignando el permiso.

- Id_Categoria: Llave foránea, corresponde a la categoría en la que se otorga el permiso.

- Tipo_Permiso: Tipo de permiso que se otorga (crear, editar, borrar).

\section{Suspensiones:}

- Id_Suspension: Llave primaria, número de identificación de la suspensión.

- Id_Usuario_Otorga: Llave foránea, corresponde al usuario que otorga la suspensión.

- Id_Usuario_Recibe: Llave foránea, corresponde al usuario que recibe la suspensión.

- Desc_Suspension: Descripción del motivo de la suspensión.

- $\quad$ Fecha_Inicio: Fecha en que se otorga la suspensión.

- Fecha_Fin: Fecha en que termina la suspensión.

Este modelo de base de datos se genera para su posterior implementación en el sistema de gestión de bases de datos de MySQL. 
El diseño gráfico del "mock-up" del sitio web de la institución educativa se dividió en secciones para facilitar la obtención de requerimientos.

Primera sección:

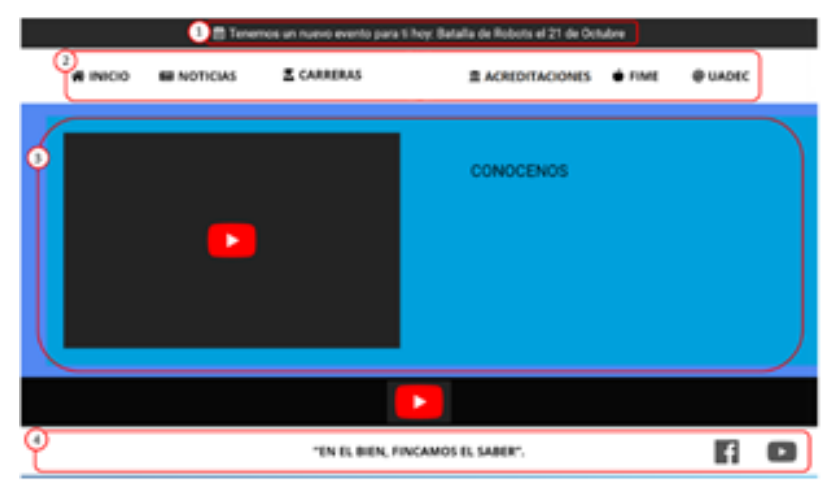

Figura 4 Primera sección, "mock-up" sitio web Fuente: Elaboración Propia

1. Listón de avisos actuales/relevantes. Muestra el aviso actual (creado más recientemente), o el aviso más relevante (el próximo en fecha de fin).

2. Listón de botones principal. Proporciona enlaces a las demás páginas del sitio, dando acceso a las funciones principales del sitio.

3. Información actual, relevante e importante. Proporciona acceso rápido a la información más reciente, la información más relevante y la información más importante para los usuarios.

4. Otros enlaces. Proporciona enlaces a sitios externos relevantes para el usuario.

Segunda sección:

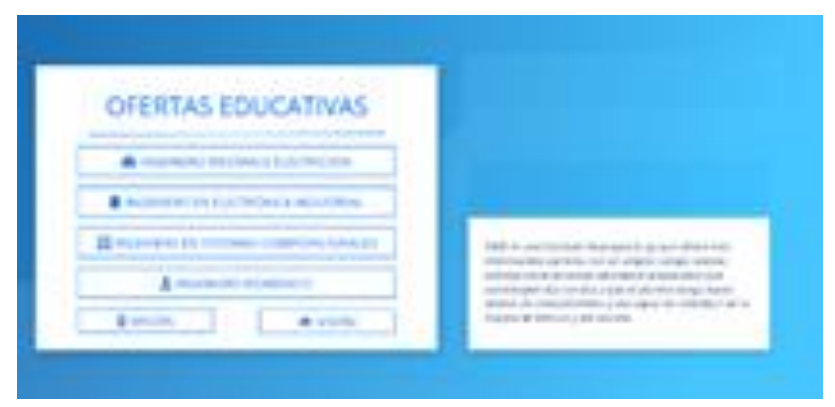

Figura 5 Segunda sección, "mock-up" sitio web Fuente: Elaboración Propia

Información general de la oferta educativa de la institución. Proporciona información general con las que cuenta, u oferta, además de enlazar a las páginas correspondientes.
Tercera sección:

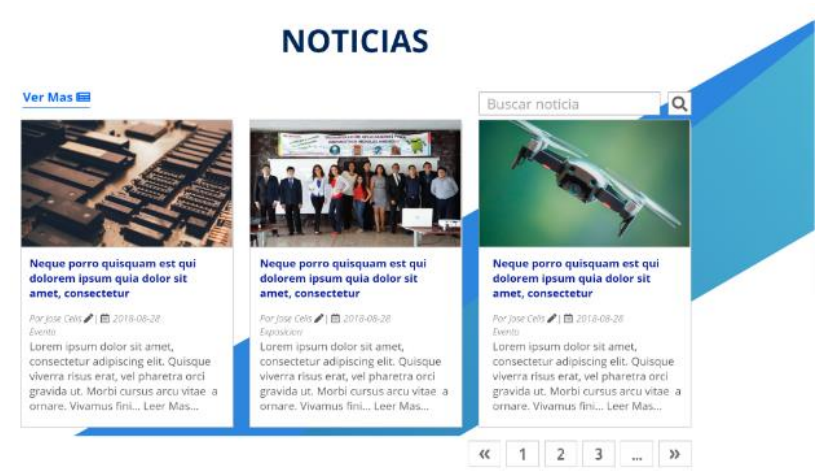

Figura 6 Tercer sección, "mock-up" sitio web Fuente: Elaboración Propia

Noticias recientes. Proporciona información general de las noticias recientes de la institución, permite buscar noticias específicas, buscar noticias anteriores, además enlaza a la página de noticias y las páginas de noticias individuales.

Cuarta sección:
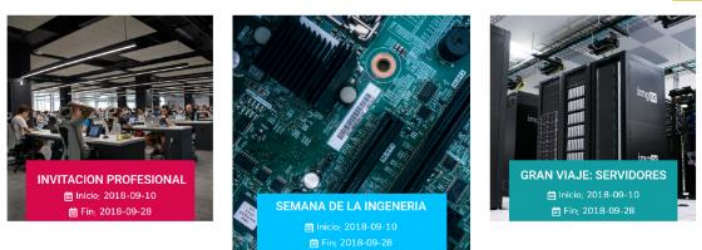

Figura 7 Cuarta sección, "mock-up" sitio web Fuente: Elaboración Propia

Eventos recientes/relevantes. Proporciona información general de los eventos recientes (creados recientemente), de los eventos que están por finalizar (fechas finales próximas), y de los eventos relevantes para la institución, además enlaza a la página de eventos y las páginas individuales de los eventos.

Quinta sección:

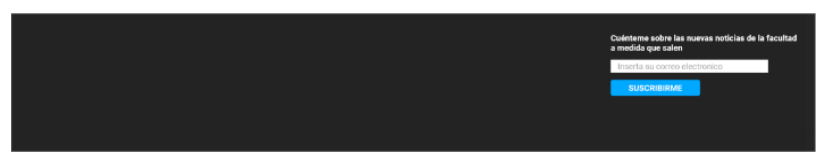

Figura 8 Quinta sección, "mock-up" sitio web Fuente: Elaboración Propia

Contactos u suscripción. Muestra la información de contacto de la información y permite suscribirse al sitio para recibir información directa de noticias, eventos, avisos y otros cambios realizados al sitio.

VÁZQUEZ-DE LOS SANTOS, Laura Cristina, CORTES-MORALES, Griselda, VALDEZ-MENCHACA, Alicia Guadalupe y MARTÍNEZPERALES, Diego Arnulfo. Diseño de un sitio web dinámico para institución educativa. Revista de Tecnologías de la Información. 2019 


\section{Resultados}

En este artículo se muestran dos etapas del proceso de desarrollo de software clásico: el análisis de requerimientos y el diseño del modelo de datos. Los resultados obtenidos se apegan a estas etapas y posteriormente se pretende sean utilizados para llevar a cabo el resto del desarrollo.

Los resultados obtenidos en la etapa de análisis fueron los requerimientos lógicos del sitio, las funciones que se requieren para poder dar servicio a un sitio web informativo y la información que se requiere recopilar para la correcta función de este. A partir de los resultados obtenidos en la primera etapa se procedió al diseño del modelo de datos.

Los resultados obtenidos del diseño del modelo de datos fueron los modelos lógicos y de base de datos con los cuales se creó el script para la creación de la base de datos dinámica, la cual se utilizará para almacenar toda la información pertinente al sitio web de la institución.

Además, como parte del diseño de modelo de datos se crearon los scripts para el sistema CRUD, el cual es utilizado para dar al sitio web interacción con la base de datos, garantizando que en todo momento se conozca la manera en que se manipulan los datos, que los datos se puedan manipular fácilmente por el personal de la facultad, y que el mantenimiento del sitio sea más fácil de realizar, todo esto sin necesidad de tener conocimiento del funcionamiento interno del sitio.

De esta manera los resultados de este trabajo de tesis sirven para garantizar la correcta funcionalidad de la base de datos y diseño del sitio web dinámico de la institución educativa permitió agregar, editar y actualizar la información de manera fácil.

\section{Agradecimiento}

Se agradece el apoyo proporcionado por la Universidad Autónoma de Coahuila para el desarrollo y publicación de este artículo.

\section{Conclusiones}

Cuando se trata del desarrollo de software se tiende a utilizar procesos de desarrollo que den sobre todo rapidez de resultados, ya que el mundo del software se encuentra en constante cambio y las necesidades de software surgen de un momento a otro, esto lleva al desarrollo de sistemas cuya vida útil es corta.

El propósito de este trabajo es el de encontrar un punto medio entre un desarrollo veloz, y un desarrollo que de longevidad al software creado. Parte de esto incluye la documentación de tanto el conocimiento utilizado para llevar a cabo las etapas de desarrollo, las necesidades expresadas por los usuarios finales del sistema (análisis de requerimientos), los diseños lógicos del sistema y el código generado con el cual se llevarán a cabos las etapas posteriores de desarrollo.

\section{Referencias}

Arias, A. and Durango, A. (2016). Ingeniería y Arquitectura del Software: $2^{\mathrm{a}}$ Edición. ITCampusAcademy.

Calero, C., Piattini, M. and Moraga, M. (2010). Calidad del producto y proceso software. Madrid: RA-MA.

Genero, M. and Piattini, M. (2014). Métodos de investigación en ingeniería del software. Madrid: RA-MA.

Pantaleo, G. and Rinaudo, L. (2016). Ingeniería de Software. México: Alfaomega.

Piñeiro Gómez, J. M. (2014). Diseño de bases de datos relacionales. UF2175 (Ed. rev.). Madrid, España: Paraninfo.

Pressman, R. S. (2010). Ingeniería del software. Un enfoque práctico. ( $7^{\mathrm{a}}$ ed.). México, México: McGraw Hill. 